\title{
Lésions néoplasiques précoces du tube digestif : introduction
}

\section{Early Neoplastic Lesions of the Gastrointestinal Tract: Introduction}

\section{Barthet}

(C) Lavoisier SAS 2017

Les discordances en faux-positifs ou faux-négatifs atteignent 30 à $50 \%$ des biopsies contrôlées par mucosectomie ou dissection sous-muqueuse, pour l'endobrachyœsophage, les adénomes duodénaux ou les ampullomes. Dans trois séries de notre équipe, dont deux présentées au cours de ces JFHOD 2017, cette discordance était confirmée avec également des taux de variabilité interobservateurs déficients, y compris chez les experts. Concernant la dysplasie de haut grade en cas d'endobrachyœsophage, nous ne confirmions la DHG initialement diagnostiquée sur biopsie que dans $52 \%$ des cas de mucosectomie. Le coefficient kappa de la relecture entre experts était faible $(0,2-0,5)$. La discordance entre biopsies et pièces opératoires atteignait aussi un quart des ampullomes. Dans notre série de 61 ampullectomies endoscopiques, $26 \%$ n'avaient pas de tumeur sur la pièce opératoire, ce taux variant de 24 à $36 \%$ dans la littérature. Enfin, dans la série bicentrique de 134 mucosectomies duodénales pour adénome, $32 \%$ des lésions étaient sousévaluées par la biopsie initiale et 10,6\% surévaluées. De plus, les biopsies n'avaient jamais diagnostiqué un adénocarcinome présent chez $8 \%$ des patients.

Le débat est donc amorcé. Quelle confiance avoir dans les biopsies avant résection et peut-on indiquer une résection endoscopique sur leur résultat? 